\title{
Respiratory Support in Patients With Amyotrophic Lateral Sclerosis
}

Despite advances in the medical therapy of amyotrophic lateral sclerosis (ALS), the management of ALS remains predominantly supportive, with a primary focus on the respiratory system, including support of ventilation and clearance of secretions. Two papers in this issue of RESPIRATORY CARE address the respiratory care of patients with ALS. ${ }^{1,2}$

\section{SeE the Original Studies \\ on PAges 1424 ANd 1433}

To contrast the impact of pharmacologic and respiratory interventions, riluzole, the only drug documented to prolong survival in ALS, prolongs median survival by 2-3 months with no clear subjective benefits, ${ }^{3}$ whereas noninvasive ventilation (NIV) prolonged median survival by 7-12 months and improved quality of life in a randomized trial, ${ }^{4}$ and cohort studies. ${ }^{5,6}$ Several challenges remain in the application of NIV in patients with ALS, including the timing of initiation, whether or not it favorably alters the course of diaphragm impairment, and underutilization.

Current criteria for Medicare coverage of NIV in patients with ALS include any of: $\mathrm{FVC}<50 \%$, maximum inspiratory pressure $<60 \mathrm{~cm} \mathrm{H}_{2} \mathrm{O}, \mathrm{S}_{\mathrm{pO}_{2}} \leq 88 \%$ for at least 5 continuous minutes, or a $\mathrm{P}_{\mathrm{aCO}}$ of $>45 \mathrm{~mm} \mathrm{Hg} .{ }^{7}$ The Practice Parameters update of the American Academy of Neurology added a sniff nasal pressure $<40 \mathrm{~cm} \mathrm{H}_{2} \mathrm{O}$ and orthopnea as additional criteria that may justify initiation of NIV. ${ }^{8}$ While earlier initiation of NIV may have advantages, there are difficulties in achieving adherence in patients who are not symptomatic with orthopnea or dyspnea. ${ }^{9,10}$ A stronger argument for earlier initiation of NIV could be made if the intervention favorably modified the course of the disease, and there may be a scientific rationale for a favorable effect of NIV on the rate of decline of lung function.

For instance, in diseases of the anterior horn cells such as post-polio syndrome and ALS, sprouting from surviving axons reinnervates muscle fibers previously supported by the destroyed anterior horn cells, resulting in a larger muscle mass innervated by a smaller pool of motor neurons (enlarged macro-motor units). In ALS there is evidence of reinnervation leading to an increase in muscle fibers innervated by a single motor neuron, and of conduction defects at newly formed reinnervated neuromuscular junctions. ${ }^{11}$ These macro-motor units may be susceptible to failure due to drop out of the chronically overloaded lower motor neurons, loss of their larger subtended muscle mass, and dysfunction of the new neuromuscular junction. If NIV can reduce diaphragmatic muscle overload and reduce macro-motor unit drop-out, then it may have a favorable effect on the course of the disease. In several studies NIV has been proposed to decrease the rate of decline of lung function, ${ }^{4,6,12}$ but another study showed that, while the rate of lung function decline slows over time, this slowing was independent of initiation of NIV. ${ }^{5}$

There may still be advantages to earlier NIV initiation that are unrelated to a modification of the progress of the disease. For instance, early initiation improves adherence, ${ }^{13-15}$ and may therefore be a consideration for patients with expected adherence issues, such as those with bulbar symptoms. Otherwise, outcome studies regarding earlier initiation of NIV are limited. In a randomized trial, early initiation of NIV at the onset of nocturnal desaturation increased the vitality subscale of the 36-item Medical Outcomes Study Short Form questionnaire, compared to standard initiation at an FVC of $<50 \%$ of predicted. ${ }^{14}$ In a prospective study of early NIV at the onset of nocturnal desaturation $(>15$ desaturations/h), the mean survival was 11 months longer, compared to a historical control group in which NIV was started for daytime hypoxemia or hypercarbia $\left(\mathrm{P}_{\mathrm{aO}}<80 \mathrm{~mm} \mathrm{Hg}\right.$, with or without $\mathrm{P}_{\mathrm{aCO}_{2}}$ $>45 \mathrm{~mm} \mathrm{Hg}) .{ }^{15} \mathrm{In}$ a retrospective cohort, early initiation of NIV, at an FVC $\geq 65 \%$ of predicted, was associated with a significant increase in the median time from ALS diagnosis to death, compared to initiation from an FVC of $<65 \%$ of predicted (2.7 y vs $1.8 \mathrm{y}){ }^{16}$

Improved survival and improved quality of life are now well established benefits of NIV, whether the initiation of NIV is "late" (hypercapnia and FVC $38-45 \%$ of predicted at initiation), ${ }^{17}$ or "early" (FVC $49-56 \%$ of predicted at initiation). ${ }^{4}$ Therefore, while the optimal timing of NIV initiation is an important consideration, it is not as important as ensuring that those who qualify for it, based on current criteria, are offered the intervention. Unfortunately, only $21 \%$ of patients with ALS receive NIV when they would qualify for it, based on existing guidelines. ${ }^{18}$ The study by Vitacca et al in this issue of the Journal elegantly 
demonstrates the importance of a referral to specialized pulmonary care, where the reported use of NIV and assisted-cough techniques are substantially higher. ${ }^{2}$ However, they also document substantial practice variability in their survey of Italian pneumology units that treat patients with neuromuscular disease. The majority of these centers initiated NIV for diurnal hypercapnia or for nocturnal desaturation, and only $67 \%$ of the respondents considered a sitting FVC of $<50 \%$ of predicted, or a reduction in maximum inspiratory pressure to initiate NIV. Moreover, only $39 \%$ of the centers considered clinical symptoms as criteria to start NIV. ${ }^{2}$ Although these answers may reflect lack of knowledge, disagreement on guidelines, or simply practice variation, it may lead to patients not receiving therapy when it is indicated.

An additional challenge is that not all patients tolerate NIV, due to factors that may include the presence of bulbar symptoms, ${ }^{13,17}$ lower vital capacity at initiation, ${ }^{13}$ or frontotemporal dementia. ${ }^{19}$ Still, NIV improved symptoms (without a survival advantage) in patients with severe bulbar impairment, in the randomized trial by Bourke et al, ${ }^{4}$ and patients with moderate to severe bulbar symptoms may still have a survival advantage if they are adherent to NIV. ${ }^{17}$ Thus, achieving NIV tolerance has important outcome implications.

In one study, only $32 \%$ of ALS patients were adherent to NIV in the presence of moderate to severe bulbar symptoms, compared to $64 \%$ in their absence. ${ }^{5}$ In contrast, the study by Vandenberghe et al in this issue, which assessed a cohort of ALS patients for clinical predictors of NIV tolerance, confirms bulbar involvement as a predictor of NIV intolerance, but also documents a much higher overall NIV tolerance, of $75 \% .^{1}$ There may be multiple reasons for this difference, including the criteria defining adherence and the chronological difference between the studies. However, initiation of NIV is done in in-patient pulmonary wards in Europe, compared to out-patient clinics in the United States. Those dedicated units may improve NIV tolerance with the use of different masks, devices, and counseling.

Vandenberghe and colleagues found that the accumulation of secretions (as assessed via physical exam) was associated with increased NIV intolerance (odds ratio 11.5) and with a nonsignificant, but perhaps clinically relevant, decreased total time use of NIV. While bulbar involvement was also predictive of NIV intolerance, the pooling of secretions was more common ( $15 \%$ vs $5 \%$ ) in patients without bulbar involvement (as assessed by clinical scores). ${ }^{1}$ The pooling of secretions in the airway speaks of the integrity of the bulbar innervations, and Vandenberghe et al have described another way to detect bulbar impairment.

We lack an objective marker of bulbar function, and most studies have used subscores of the ALS Functional Rating Scale (ALSFRS-R) and the Norris scale. ${ }^{20,21}$ Those scores' cutoff points are arbitrary, and no study has defined a cutoff that accurately reflects an objectively measured clinical impairment. For example, in a study that assessed swallowing with scintigraphy, the ALSFRS-R bulbar score correlated with the degree of swallowing, but swallowing dysfunction was present even at the highest scores (less symptoms). ${ }^{22}$ The evaluation of bulbar function remains subjective as a clinical assessment, where some clinical tests (swallowing and speech) and patient recall define the level of bulbar involvement. This is in contrast to the evaluation of ventilation, where several objective functional markers are followed through time. Of all the routine respiratory tests ordered, peak cough flow is the only test that reflects bulbar function in addition to inspiratory and expiratory muscle function. The difference between peak cough flow and peak expiratory flow has thus been proposed to measure bulbar involvement. ${ }^{23}$ We need a more accurate way to detect and quantify the level of bulbar involvement. ${ }^{24,25} \mathrm{~A}$ specific test of bulbar function may help to recognize the group of patients at higher risk of NIV failure, at need for early initiation of mechanical cough assistance, or will simply help to better classify/quantify bulbar involvement. ${ }^{26}$

An alternative or complementary option to NIV for the support of inspiratory function is available. Diaphragm pacing was approved by the Food and Drug Administration in September 2011 as a humanitarian use device for patients with ALS. This designation was based on evidence that the device imparted probable benefits that outweigh the risk of injury. The exact indications for the device and firm evidence of benefit need to be established. For instance, the intended use of the device is for individuals with a stimulable diaphragm, in association with evidence of chronic hypoventilation, and an FVC $\geq 45 \%$ of predicted. In view of the upper and lower motor neuron distribution of ALS, the requirement for a stimulable diaphragm would require a predominance of upper motor neuron involvement and a relatively preserved lower motor neuron function. There is focality of disease distribution, with a normal distribution of lower motor loss ranging from $8 \%$ to $90 \%, 27,28$ providing a window of opportunity for diaphragm pacing in individuals with more preserved lower motor neuron function of the diaphragm. Currently, this is assessed by demonstration of voluntary contraction of the diaphragm by fluoroscopy, ultrasound of the diaphragm (documenting thickening with inspiration), or phrenic nerve conduction studies. ${ }^{29}$ There is now extensive experience in some centers, and the reported advantages include more rapid extubation after implantation with the use of the device, improved respiratory compliance, delayed need for ventilatory support (by up to 24 months), and improved sleep quality. ${ }^{30,31}$ More studies are necessary to confirm these results. Interestingly, diaphragm pacing and NIV may complement each other, as noninvasive 
devices can respond to the stimulus from the diaphragm pacer, and positive pressure can compensate for the potential of upper-airway obstruction that can occur with negative airway pressure from diaphragm pacing. ${ }^{32}$

The management of secretions poses a particular challenge in patients with bulbar disease. Not only do secretions interfere with NIV, they have a predominant role in NIV intolerance in bulbar patients and pose a potential hazard for the use of NIV. The survey by Vittaca et al highlights this issue with secretion clearance in ALS: 80\% of the referrals to the Italian pulmonology units were for secretion management, in contrast to $6 \%$ for patients with other neuromuscular diseases. ${ }^{2}$ In the same line, Vandenberghe's protocol focused on control of secretion pooling to improve NIV adherence. ${ }^{1}$ They suggest that routine assessment for pooled secretions could help identify patients at risk for NIV intolerance. Our approach to these patients is to implement appropriate secretion clearance techniques, then offer NIV, possibly earlier in the course of the disease, since earlier initiation may facilitate adherence. ${ }^{13-15}$

Secretion clearance with manually assisted and mechanically assisted cough is also life-prolonging. Peak cough flow can be enhanced by abdominal thrust, air stacking, use of NIV, or insufflation/exsufflation. ${ }^{33}$ Cough augmentation enhances secretion clearance, reduced hospitalizations and respiratory infections, and improves survival. ${ }^{34-37}$ However, the data supporting its use are mainly from observational studies, expert opinion, and retrospective studies.

Finally, if the patient is intolerant of NIV or the respiratory failure persists despite all support, the question is when to place a tracheostomy. Some studies have demonstrated prolonged survival and similar quality of life to $\mathrm{NIV}$, at the cost of an increase in respiratory infections. ${ }^{38,39}$ Questions remain regarding the optimal timing for tracheostomy placement, whether a cuffed or uncuffed tube should be used, and the ventilation mode. The survey by Vitacca et al highlights the paucity of information: $64 \%$ of the responding centers felt that tracheostomy was a poor prognostic factor, the majority considered its main indication to be failure to wean from mechanical ventilation, and $10 \%$ of the centers never offer it as an option. ${ }^{2}$ This is a sample of the plethora of views and concepts physicians hold toward tracheostomy in patients with ALS.

The authors have disclosed no conflicts of interest.

Correspondence: Loutfi S Aboussouan MD, Respiratory Institute, Cleveland Clinic, A90, 9500 Euclid Avenue, Cleveland OH 44195. E-mail: abouss1@ccf.org.

DOI: $10.4187 /$ respcare. 02710
Perhaps these views are changing in the same way our views and attitudes toward NIV have changed. ${ }^{40}$ In the past, the care of these patients was done at a few medical centers, with one physician leading the way. This expert dealt with all the neuromuscular patients, using different techniques and criteria for care. In recent years, as NIV and cough-assist devices became ubiquitous, the practice patterns have changed, and practice parameters have been published. ${ }^{8}$ More centers and physicians are caring for patients with neuromuscular diseases and implementing supportive respiratory interventions. The impact on survival is becoming apparent. Specifically, data from the placebo arms of 16 randomized trials published from 1994 to 2008 show that the survival of ALS patients has improved, even without improvements in measures of function. ${ }^{41}$ The nihilistic approach continues to wane as more patients with progressive neuromuscular diseases live longer, with better quality of life, and in their homes. While there has been substantial progress, until more definitive treatments for ALS are found, much more remains to be done in the supportive care of our ALS patients.

\section{Loutfi S Aboussouan MD Eduardo Mireles-Cabodevila MD Respiratory Institute Cleveland Clinic Cleveland, Ohio}

\section{REFERENCES}

1. Vandenberghe N, Vallet AE, Petitjean T, Le Cam P, Peysson S, Guérin $\mathrm{C}$, et al. Absence of airway secretion accumulation predicts tolerance of noninvasive ventilation in subjects with amyotrophic lateral sclerosis. Respir Care 2013;58(9):1424-1432.

2. Vitacca M, Vianello A, on behalf of the Scientific Group on Respiratory Intensive Care of the Italian Association of Hospital Pulmonologists. Respiratory outcomes of patients with amyotrophic lateral sclerosis: an Italian nationwide survey. Respir Care 2013;58(9): 1433-1441.

3. Miller RG, Mitchell JD, Lyon M, Moore DH. Riluzole for amyotrophic lateral sclerosis (ALS)/motor neuron disease (MND). Cochrane Database Syst Rev 2007;(1):CD001447.

4. Bourke SC, Tomlinson M, Williams TL, Bullock RE, Shaw PJ, Gibson GJ. Effects of non-invasive ventilation on survival and quality of life in patients with amyotrophic lateral sclerosis: a randomised controlled trial. Lancet Neurol 2006;5(2):140-147.

5. Aboussouan LS, Khan SU, Banerjee M, Arroliga AC, Mitsumoto H. Objective measures of the efficacy of noninvasive positive-pressure ventilation in amyotrophic lateral sclerosis. Muscle Nerve 2001; 24(3):403-409.

6. Kleopa KA, Sherman M, Neal B, Romano GJ, Heiman-Patterson T. BiPAP improves survival and rate of pulmonary function decline in patients with ALS. J Neurol Sci 1999;164(1):82-88.

7. Clinical indications for noninvasive positive pressure ventilation in chronic respiratory failure due to restrictive lung disease, COPD, and nocturnal hypoventilation: a consensus conference report. Chest 1999; 116(2):521-534. 


\section{Respiratory Support in Patients With Amyotrophic Lateral Sclerosis}

8. Miller RG, Jackson CE, Kasarskis EJ, England JD, Forshew D, Johnston W, et al. Practice parameter update: the care of the patient with amyotrophic lateral sclerosis: drug, nutritional, and respiratory therapies (an evidence-based review): report of the Quality Standards Subcommittee of the American Academy of Neurology. Neurology 2009;73(15):1218-1226.

9. Bourke SC, Bullock RE, Williams TL, Shaw PJ, Gibson GJ. Noninvasive ventilation in ALS: indications and effect on quality of life. Neurology 2003;61(2):171-177.

10. Jackson CE, Lovitt S, Gowda N, Anderson F, Miller RG. Factors correlated with NPPV use in ALS. Amyotroph Lateral Scler 2006; $7(2): 80-85$.

11. Yuen EC, Olney RK. Longitudinal study of fiber density and motor unit number estimate in patients with amyotrophic lateral sclerosis. Neurology 1997;49(2):573-578.

12. Schmidt EP, Drachman DB, Wiener CM, Clawson L, Kimball R, Lechtzin N. Pulmonary predictors of survival in amyotrophic lateral sclerosis: use in clinical trial design. Muscle Nerve 2006;33(1): 127-132.

13. Gruis KL, Brown DL, Schoennemann A, Zebarah VA, Feldman EL. Predictors of noninvasive ventilation tolerance in patients with amyotrophic lateral sclerosis. Muscle Nerve 2005;32(6):808-811.

14. Jackson CE, Rosenfeld J, Moore DH, Bryan WW, Barohn RJ, Wrench $\mathrm{M}$, et al. A preliminary evaluation of a prospective study of pulmonary function studies and symptoms of hypoventilation in ALS/MND patients. J Neurol Sci 2001;191(1-2):75-78.

15. Pinto A, de Carvalho M, Evangelista T, Lopes A, Sales-Luis L. Nocturnal pulse oximetry: a new approach to establish the appropriate time for non-invasive ventilation in ALS patients. Amyotroph Lateral Scler Other Motor Neuron Disord 2003;4(1):31-35.

16. Lechtzin N, Scott Y, Busse AM, Clawson LL, Kimball R, Wiener $\mathrm{CM}$. Early use of non-invasive ventilation prolongs survival in subjects with ALS. Amyotroph Lateral Scler 2007;8(3):185-188.

17. Aboussouan LS, Khan SU, Meeker DP, Stelmach K, Mitsumoto H. Effect of noninvasive positive-pressure ventilation on survival in amyotrophic lateral sclerosis. Ann Intern Med 1997;127(6):450-453.

18. Miller RG, Anderson F, Brooks BR, Mitsumoto H, Bradley WG, Ringel SP. Outcomes research in amyotrophic lateral sclerosis: lessons learned from the amyotrophic lateral sclerosis clinical assessment, research, and education database. Ann Neurol 2009;65 (Suppl 1):S24-S28.

19. Olney RK, Murphy J, Forshew D, Garwood E, Miller BL, Langmore $\mathrm{S}$, et al. The effects of executive and behavioral dysfunction on the course of ALS. Neurology 2005;65(11):1774-1777.

20. Cedarbaum JM, Stambler N, Malta E, Fuller C, Hilt D, Thurmond B, Nakanishi A. The ALSFRS-R: a revised ALS functional rating scale that incorporates assessments of respiratory function. BDNF ALS Study Group (Phase III). J Neurol Sci 1999;169(1-2):13-21.

21. Norris FH Jr, Calanchini PR, Fallat RJ, Panchari S, Jewett B. The administration of guanidine in amyotrophic lateral sclerosis. Neurology 1974;24(8):721-728.

22. Fattori B, Grosso M, Bongioanni P, Nacci A, Cristofani R, AlSharif A, et al. Assessment of swallowing by oropharyngoesophageal scintigraphy in patients with amyotrophic lateral sclerosis. Dysphagia 2006;21(4):280-286.

23. Suarez AA, Pessolano FA, Monteiro SG, Ferreyra G, Capria ME, Mesa L, et al. Peak flow and peak cough flow in the evaluation of expiratory muscle weakness and bulbar impairment in patients with neuromuscular disease. Am J Phys Med Rehabil 2002;81(7): 506-511.

24. Kuhnlein P, Gdynia HJ, Sperfeld AD, Lindner-Pfleghar B, Ludolph AC, Prosiegel M, Riecker A. Diagnosis and treatment of bulbar symptoms in amyotrophic lateral sclerosis. Nat Clin Pract Neurol 2008;4(7):366-374.

25. Yunusova Y, Green JR, Wang J, Pattee G, Zinman L. A protocol for comprehensive assessment of bulbar dysfunction in amyotrophic lateral sclerosis (ALS). J Vis Exp 2011;(48). DOI: 10.3791/2422. http://www.jove.com/video/2422/a-protocol-for-comprehensiveassessment-bulbar-dysfunction. Accessed July 3, 2013.

26. Sancho J, Servera E, Diaz J, Marin J. Predictors of ineffective cough during a chest infection in patients with stable amyotrophic lateral sclerosis. Am J Respir Crit Care Med 2007;175(12):1266-1271.

27. Ravits J, Paul P, Jorg C. Focality of upper and lower motor neuron degeneration at the clinical onset of ALS. Neurology 2007;68(19): 1571-1575.

28. Ravits J, Laurie P, Fan Y, Moore DH. Implications of ALS focality: rostral-caudal distribution of lower motor neuron loss postmortem. Neurology 2007;68(19):1576-1582.

29. McCool FD, Tzelepis GE. Dysfunction of the diaphragm. N Engl J Med 2012;366(10):932-942.

30. Gonzalez-Bermejo J, Morelot-Panzini C, Salachas F, Redolfi S, Straus C, Becquemin $\mathrm{MH}$, et al. Diaphragm pacing improves sleep in patients with amyotrophic lateral sclerosis. Amyotroph Lateral Scler 2012;13(1):44-54.

31. Onders RP, Elmo M, Khansarinia S, Bowman B, Yee J, Road J, et al. Complete worldwide operative experience in laparoscopic diaphragm pacing: results and differences in spinal cord injured patients and amyotrophic lateral sclerosis patients. Surg Endosc 2009; 23(7):1433-1440.

32. Nishino T. Problems associated with diaphragm pacing. Respiration 2002;69(1):12-13.

33. Senent C, Golmard JL, Salachas F, Chiner E, Morelot-Panzini C, Meninger $\mathrm{V}$, et al. A comparison of assisted cough techniques in stable patients with severe respiratory insufficiency due to amyotrophic lateral sclerosis. Amyotroph Lateral Scler 2011;12(1):26-32.

34. Chatwin M, Simonds AK. The addition of mechanical insufflation/ exsufflation shortens airway-clearance sessions in neuromuscular patients with chest infection. Respir Care 2009;54(11):1473-1479.

35. Vitacca M, Paneroni M, Trainini D, Bianchi L, Assoni G, Saleri M, et al. At home and on demand mechanical cough assistance program for patients with amyotrophic lateral sclerosis. Am J Phys Med Rehabil 2010;89(5):401-406.

36. Moran FC, Spittle A, Delany C, Robertson CF, Massie J. Effect of home mechanical in-exsufflation on hospitalisation and life-style in neuromuscular disease: a pilot study. J Paediatr Child Health 2013; 49(3):233-237.

37. Miske LJ, Hickey EM, Kolb SM, Weiner DJ, Panitch HB. Use of the mechanical in-exsufflator in pediatric patients with neuromuscular disease and impaired cough. Chest 2004;125(4):1406-1412.

38. Sancho J, Servera E, Banuls P, Marin J. Prolonging survival in amyotrophic lateral sclerosis: efficacy of noninvasive ventilation and uncuffed tracheostomy tubes. Am J Phys Med Rehabil 2010;89(5): 407-411.

39. Vianello A, Arcaro G, Palmieri A, Ermani M, Braccioni F, Gallan F, et al. Survival and quality of life after tracheostomy for acute respiratory failure in patients with amyotrophic lateral sclerosis. J Crit Care 2011;26(3):329.e7-e14.

40. Bach JR, Goncalves MR, Hon A, Ishikawa Y, De Vito EL, Prado F, Dominguez ME. Changing trends in the management of end-stage neuromuscular respiratory muscle failure: recommendations of an international consensus. Am J Phys Med Rehabil 2013;92(3):267-277.

41. Qureshi M, Schoenfeld DA, Paliwal Y, Shui A, Cudkowicz ME. The natural history of ALS is changing: improved survival. Amyotroph Lateral Scler 2009;10(5-6):324-331. 